\title{
Konzepte zur Integration von Flexibilitäten in Wohn- und Nichtwohngebäuden in ein dynamisches Lastmanagement
}

\author{
M. Becker@, T. Müller, M. H. Forchheim, D. Cano-Tirado, M. Zdrallek
}

Dieser Artikel stellt die Konzepte zweier Add-on-Systeme für Verteilnetzautomatisierungssysteme vor, deren Ziel die Nutzung neuer Netzaktoren, wie die Ladeinfrastruktur für Elektrofahrzeuge, elektrische Wärmepumpen und Batteriespeicher, als Flexibilitäten für ein dynamisches und marktorientiertes Netzengpassmanagement ist.

Die entwickelten Konzepte legen dabei jeweils den Fokus auf den regulierten Bereich des Netzbetreibers (Fokus des Projektes ElCiN) und den freien Marktbereich der Anschlussnehmer (Fokus des Projektes InFleX), ohne die jeweiligen Anforderungen des anderen Bereichs außer Acht zu lassen.

Das im ElCiN-Projekt entwickelte Gesamtkonzept, welches den zellularen Ansatz des VDE umsetzt (VDE Verband der Elektrotechnik Elektronik Informationstechnik e.V., Zellulares Energiesystem: Ein Beitrag zur Konkretisierung des zellularen Ansatzes mit Handlungsempfehlungen, https://www.vde.com/resource/blob/1884494/98f96973fcdba70777654d0f40c179e5/studie---zellularesenergiesystem-data.pdf 2019), ermöglicht ein vollautomatisiertes und marktorientiertes Netzengpassmanagement. Dabei wurde ein Datenaustausch zwischen reguliertem Bereich und Marktbereich definiert, der einzelne neue Netzaktoren, aber auch in Zellen zusammengefasste und ggf. optimierte Netzaktoren, in das Netzengpassmanagement integrieren kann.

Das InFleX-Projekt beschäftigt sich mit der Hebung der Flexibilitäten für (Nicht-) Wohngebäude und deren energetischen Optimierung und Flexibilitätsbereitstellung für Verteilnetzautomatisierungssysteme.

Beide entwickelten Konzepte sowie die daraus entstandenen Systeme können grundsätzlich zu einem Gesamtsystem zusammengefasst werden. Ein solches Gesamtsystem setzt dann nicht nur den zellularen Ansatz des VDE um, sondern tut dies unter Berücksichtigung der Anforderungen und Interessen des Netzbetreibers sowie des Anschlussnehmers.

Schlüsselwörter: Netzautomatisierung; dynamisches Lastmanagement; Lastmanagement; Flexibilitäten

\section{Concepts for integrating flexibilities into dynamic load management for residential and non-residential buildings.}

This article presents the concepts of two add-on systems for smart grids, which aim to integrate new actuators, such as the charging infrastructure for electric vehicles, electric heat pumps, and battery storage systems, as flexibility options for dynamic and marketoriented network congestion management.

The developed concepts focus on the regulated sector of the grid operator (the EICiN project's focus) and on the free-market sector of the energy customers (the InFleX project's focus), taking the requirements of both sectors into account.

The overall concept developed in the course of the EICiN project, which implements the cellular approach of the VDE (VDE Verband der Elektrotechnik Elektronik Informationstechnik e.V., Zellulares Energiesystem: Ein Beitrag zur Konkretisierung des zellularen Ansatzes mit Handlungsempfehlungen, https://www.vde. com/resource/blob/1884494/98f96973fcdba70777654d0f40c179e5/studie--zellulares-energiesystem-data.pdf 2019), enables a fully automated and market-oriented network congestion management. Data exchange between the regulated sector and the market sector was defined, which can integrate individual new grid actuators as well as grid actuators that are pooled and potentially optimized in cells into the network congestion management.

The InFleX project deals with enhancing the flexibilities for (non-) residential buildings and their energetic optimization as well as with providing flexibilities for smart grids.

Both developed concepts and the systems resulting from them can be combined to create an overall system. Such a system implements the cellular approach of the VDE without disregarding the requirements and interests of the grid operator and the customer.

Keywords: smart grid; dynamic load management; load management; flexibilities

\section{Einleitung}

Die Klimaziele der Bundesrepublik Deutschland sehen vor, dass bis 2030 die Treibhausgasemissionen um 55 Prozent im Vergleich zu 1990 sinken sollen und bis 2050 die Treibhausgasneutralität erreicht wird [1]. Die zur Erreichung der Klimaziele notwendige Energiewende in Deutschland führt nicht nur zu Veränderungen in der Energieerzeugung, weg von fossilen Energieträgern hin zu Erneuerba-
Becker, Michael, Bergische Universität Wuppertal, Rainer-Gruenter-Straße 21, 42119 Wuppertal, Deutschland (E-Mail: michael.becker@uni-wuppertal.de); Müller, Tobias, Bergische Universität Wuppertal, Wuppertal, Deutschland; Forchheim, Maximilian Hendrik, Bergische Universität Wuppertal, Wuppertal, Deutschland; Cano-Tirado, David, Bergische Universität Wuppertal, Wuppertal, Deutschland; Zdrallek, Markus, Bergische Universität Wuppertal, Wuppertal, Deutschland 
ren Energien, sondern auch zu Veränderungen in den Bereichen der Mobilität (Hochlauf der Elektromobilität) und der Wärmeversorgung (Hochlauf von elektrischen Wärmepumpen) [2]

Die Einbindung von Erneuerbaren Energien wie Photovoltaik- (PV) und Windkraftanlagen in die Verteilnetze, die für diese Versorgungssituation nicht ausgelegt sind, kann jedoch zu Netzengpässen wie Spannungsbandverletzungen oder Betriebsmittelüberlastungen führen [3]. Gleichwohl sind neue zusätzliche Lasten, wie Ladeinfrastruktur für Elektrofahrzeuge (LIS) und elektrische Wärmepumpen (WP), in der Vergangenheit nicht in der Netzplanung berücksichtigt worden. Der starke Zubau dieser Lasten kann wiederum ebenfalls zu Netzengpässen führen [4, 5].

Diese neuen Lasten sind jedoch nicht nur neue zusätzliche Lasten, sondern stehen dem Verteilnetz auch im Rahmen ihrer technischen Möglichkeiten als Flexibilitäten zur Verfügung [6]. Durch Einbindung der Flexibilitäten in ein dynamisches Lastmanagement können temporäre Netzengpässe behoben, der Verteilnetzausbau verzögert oder ggf. vermieden und der Ausnutzungsgrad der volatilen Energieerzeugung durch PV- und Windkraftanlagen verbessert werden, indem im Starkeinspeisefall überschüssige Energie lokal verbraucht wird

Dieser Artikel stellt die Forschungsprojekte EICiN (Electric City Neuss) und InFleX (Integration gebäudetechnischer Flexibilitäten und kombinierter Fahrzeugladeinfrastruktur in ein dezentrales Verteilnetzautomatisierungssystem) vor, die sich mit der Hebung und Nutzung netzdienlicher Flexibilitäten im Verteilnetz beschäftigen.

In Abschn. 2 wird zunächst das im EICiN-Projekt erarbeitete Konzept zum Aufbau des ElCiN-Regelungssystems beschrieben. Dieses Konzept beschäftigt sich mit der Hebung von Flexibilitäten aus primärer Sicht des Netzbetreibers, ohne die Anforderungen des Anschlussnehmers zu vernachlässigen. Anschließend wird das InFleX-Konzept für die Nutzung gebäudespezifischer Flexibilitäten aus primärer Sicht des Anschlussnehmers bzw. Gebäudemanagers/betreibers unter Berücksichtigung der Anforderungen weitere externer Institutionen wie Netzbetreiber und Energiehändler, bzw. Aggregatoren, beschrieben.

Neben den Konzepten werden für beide Projekte auch erste Simulationsergebnisse dargestellt. Für das EICiN-Projekt werden zudem die notwendigen Konzeptanpassungen für einen durchzuführenden Feldtest beschrieben, damit das Konzept mit der aktuell am Markt verfügbaren Technik umgesetzt werden kann

In Abschn. 3 werden die Projekte gegenübergestellt und Gemeinsamkeiten sowie Unterschiede diskutiert.

Abschließend wird im 4. Abschn. ein Fazit gezogen sowie Ausblick gegeben

\section{Konzepte zur Flexibilitätshebung}

\subsection{EICiN}

\subsubsection{Einleitung und Ziel}

Im Forschungsprojekt EICiN wird ein Regelungssystem (kurz: EICiNRS) entwickelt, welches Sektorenkopplungsanlagen wie WP und LIS aber auch Batteriespeicher (elektrischen Energiespeicher; EES) als Flexibilitäten für dynamisches Lastmanagement nach dem BDEW Ampelkonzept in ein Verteilnetzautomatisierungssystem (VNA) einbindet. Das EICiN-System arbeitet dazu in der gelben Ampelphase (marktorientiert) und ergänzt dadurch klassische VNA, die in der roten Ampelphase agieren [7].

\subsubsection{Entwicklung des Regelungskonzepts}

Grundannahmen Wie der Verband der Elektrotechnik Elektronik Informationstechnik e.V. (VDE) in seinem Fachbeitrag "Zellulares Energiesystem" bereits resümierend festgestellt hat, befindet sich das deutsche Energiesystem im Wandel. Dabei ist zu erwarten, dass neben dem weiteren Ausbau dezentraler Erneuerbarer Energien auch die Sektorenkopplung, also die Verknüpfung der Sektoren Strom, Gas, Wärme und Mobilität und damit auch die Elektrifizierung der Verbrauchssektoren, weiter voranschreitet [8].

Um den zukünftigen Herausforderungen gerecht zu werden, wurde u. a. aus diesen Gründen vom VDE der zellulare Ansatz entwickelt, da zukünftig der lokale Energieausgleich auf möglichst niedriger Ebene angestrebt wird [8,9]. Der zellulare Ansatz ist die Grundlage des EICiN-Regelungskonzepts.

Die unterste Zellebene (Zellebene 5) aus Sicht des Stromnetzbetreibers stellt die Haushalts- bzw. Gewerbezelle (nachfolgend Zelle genannt) in der Niederspannung dar [8]. Hier entnimmt der Anschlussnehmer bzw. Anschlussnutzer (ASN) elektrische Energie für seine Bedürfnisse (z. B. Kühlen, Heizen, Licht, Unterhaltung).

Die über Zellebene 5 liegende Zellebene 4 ist das Niederspannungs(orts-)netz (NS-ON). Hier kann nach zellularem Ansatz ein Energieausgleich zwischen den einzelnen unterlagerten Zellen stattfinden. So könnten auf dieser Ebene mittels Flexibilitäten der einzelnen Zellen (sofern vorhanden und bereitgestellt) Netzengpässe behoben werden [8]. Da für die nachfolgenden Erarbeitungen dieses Artikels die NS-ON Ebene ausreicht, wird für weiterführende Informationen zum Aufbau des zellularen Ansatzes auf [8] verwiesen.

Unterstellt man dem ASN, dass er nach dem Wirtschaftlichkeitsprinzip handelt, wird er dezentrale Energieerzeugung und/oder Eigenbedarfsoptimierung betreiben, wenn sich für inn ein finanzieller Vorteil ergibt. Speziell die Eigenbedarfsoptimierung, beispielsweise mit einem Home-Energy-Management-System (HEMS), stellt jedoch für den Netzbetreiber einen schwer zu planenden Energieverbrauch/-überschuss dar, da der ASN die Optimierung nach seinen eigenen Bedürfnissen und Zielen durchführt

Ob ein ASN Flexibilitäten für das Energiesystem bereitstellt, hängt u. a. von seinem (weiteren) finanziellen Vorteil, der Datensicherheit seiner Kundendaten und der Kontrolle über die Flexibilität ab $[10,11]$

Rahmenbedingungen Auf Basis der beschriebenen Ausgangslage verfolgt das EICiN-Regelungskonzept den zellularen Ansatz und soll bei Netzengpässen im NS-ON (Zellebene 4) Flexibilitäten der Zellen (Zellebene 5) nutzen, um die Engpässe zu beheben. Darüber hinaus soll das Regelungskonzept folgende Anforderungen erfüllen:

1. Das zu entwickelnde EICiN-RS soll vollautomatisiert und prädiktiv Flexibilitäten zur Netzengpassbehebung einsetzen.

2. Das EICiN-RS soll in der gelben BDEW Ampelphase (marktorientiert) agieren und Netzengpässe beheben, bevor ein VNA eingreift (rote BDEW-Ampelphase)

3. Der Gesamtsystemaufbau soll Markt und regulierten Bereich klar trennen.

4. Zur Steigerung der Akzeptanz sollen die ASN jederzeit den Einsatz ihrer Flexibilitäten steuern und beeinflussen können und so die Kontrolle über ihre Anlagen behalten

5. Die unterschiedlichen Teilsysteme sollen nur die zur Netzsteuerung notwendigen Daten austauschen, sodass die Datensicherheit aufgrund von Datenminimierung erhöht wird.

6. Der Datenaustausch der Teilsysteme soll allgemeingültig gestaltet werden, sodass für alle Flexibilitäten die Daten in der gleichen Struktur ausgetauscht werden können. 
7. Das Gesamtsystem muss bereits in der Übergangsphase vom heutigen unflexiblen Energiesystem in ein vollständig flexibles Energiesystem funktionieren.

Konzeptbeschreibung Damit der Netzbetreiber prädiktiv Netzengpässe verhindern kann, benötigt er eine genaue Prognose der sich im Netz befindenden Erzeuger und Lasten. Grundsätzlich kann eine entsprechende Prognose zentral vorgenommen werden [12], beispielsweise beim Netzbetreiber, jedoch sind hier zunächst Informationen über das Verhalten des ASN nur schwer vorherzusagen und "kurzfristige Bedürfnisse" des ASN können vollständig nicht berücksichtigt werden. Um auch bspw. das kurzfristige Laden eines Elektrofahrzeugs prognostizieren zu können, bedarf es daher Echtzeitinformation des ASN.

Die gleiche Problematik, jedoch in deutlich größerem Umfang, findet bei der Ermittlung der Flexibilität eines Aktors statt. Hier kann der Netzbetreiber ebenfalls die Flexibilität selbst ermitteln, jedoch benötigt er bspw. im Falle einer WP nach Analyse im ElCiN-Projekt mindestens Anlagenmessdaten wie Vor- und Rücklauftemperatur, Speichertemperatur(en), Anlagenzustand, elektrische Leistung und Wärmeleistung des Gebäudes sowie die Anlagenparameter, u. a. die Soll-Vorlauftemperatur bzw. Heizkurve und die Zeitsteuerungen für Zirkulationspumpen etc. für eine präzise Ermittlung der Flexibilität.

In beiden Fällen (Prognose und Flexibilitätsermittlung) bedeutet dies jedoch, dass direkte Kundeninformationen dem Netzbetreiber zur Verfügung gestellt werden müssen. Gleichzeitig bleibt die Frage offen, wie sinnvoll speziell die Flexibilitätsbestimmung durch den Netzbetreiber ist. Im Forschungsprojekt EICiN wurde bei der Untersuchung der Aktoren LIS und WP deutlich, wie unterschiedlich die Systeme unterschiedlicher Hersteller (bzw. unterschiedlicher Installationen bei ANS) sind und wie aufwändig die Ermittlung der Flexibilität ist. Daher müsste von Seiten des Netzbetreibers jede individuelle Applikation analysiert und integriert werden, was mit einem hohen Aufwand bei der Implementierung und Datenhaltung verbunden ist. Will der ASN zudem noch Eigenoptimierung mit seinen Anlagen durchführen, steigt die Komplexität speziell der Flexibilitätsermittlung und damit der Aufwand beim Netzbetreiber zusätzlich an. Darüber hinaus stellen sich hier rechtliche und regulatorische Fragen, u. a. wie dies diskriminierungsfrei umgesetzt werden kann oder welche zusätzlichen Markteintrittsbarrieren für neuen Produkte in diesem Bereich entstehen könnten.

Aus den oben genannten Gründen wird daher eine Trennung von reguliertem Bereich (Zellebene 4) und Marktbereich (Zellebene 5) vorgenommen (Anforderung 3). Der Datenaustausch zwischen den Ebenen beschränkt sich dabei auf die Prognose und die Flexibilität der Zelle (von Zellebene 5 nach 4) bzw. den Flexibilitätsabruf (von Zellebene 4 nach 5). Dadurch wird zum einen die Datensicherheit erhöht, da nur die minimal notwendigen Daten ausgetauscht werden und direkte Kundendaten hier bereits bei der Prognose- und Flexibilitätsermittlung berücksichtigt wurden und somit nicht direkt erkennbar sind (Anforderung 5), zum anderen beschreibt dies einen allgemeingültigen Datenaustausch (Anforderung 6), da diese Informationen potenziell von jeder Zelle bzw. einzelnem Aktor im Netz bereitgestellt werden können.

Da die Prognoseerstellung und Flexibilitätsermittlung im Bereich des ASN geschieht, besitzt dieser weiterhin die Kontrolle über seine Anlage und kann diese zudem nach seinen Optimierungszielen in seiner Eigenoptimierung berücksichtigen. Wie oben beschrieben, sollte dies die Akzeptanz einer Flexibilitätsbereitstellung fördern (Anforderung 4).

Das Konzept sieht vor, dass das ElCiN-RS vollautomatisiert und prädiktiv auf Basis der Prognosen das Netz überwacht und prognostizierte Netzengpässe mittels bereitgestellter Flexibilitäten behebt.
Daher wird eine dauerhafte Kommunikation zwischen der NS-ON Ebene und den einzelnen Zellen benötigt. Eine minütliche Datenübertragung führt jedoch zu einem hohen Datenverkehr und ein minütlicher Abruf der Flexibilitäten ggf. zu einer stark schwankenden Fahrweise, was je nach Aktor (z. B. WP) zu erhöhtem Verschleiß führen kann [13]. Daher wurde festgelegt, dass der Zeithorizont je Prognose bzw. Flexibilität 15 Minuten beträgt. Die Prognosen werden in ein Minuten Zeitschritten und die Flexibilität für den gesamten 15 Minuten Zeitschritt jeweils 15 Minuten vor Beginn der Regelperiode an das EICiN-RS übermittelt. Das EICiN-RS wiederum verschickt drei Minuten vor dem Start der Regelperiode die benötigten Flexibilitätsabrufe an die Zellen. Der Flexibilitätsabruf gilt dann für die gesamten 15 Minuten der Regelungsperiode (Anforderung 1 und 2).

Das Konzept sieht zudem vor, dass das EICiN-RS in der gelben Ampelphase, also marktorientiert, agiert. Dazu werden mit Übermittlug der Flexibilität diese ebenfalls vom ASN bepreist. Das EICiN-RS sucht dann bei prognostizierten Netzengpässen aus allen angebotenen Flexibilitäten die kostengünstigste Lösung, um den Netzengpass zu beheben (Anforderung 2). Der Regelalgorithmus des EICiN-RS basiert im Grundsatz auf [14, 15], wonach eine Lösung des Netzengpasses von den Kosten (Fixkosten und Kosten pro geänderter Energie) sowie der Sensitivität auf den Netzengpass und somit der notwendigen Energiemenge abhängt. Im EICiN-RS werden nur Arbeitspreise (Kosten pro geänderter Energie) berücksichtigt.

Kann das EICiN-RS aufgrund von fehlenden oder nicht ausreichend zur Verfügung stehenden Flexibilitäten die prognostizierten Netzengpässe nicht vollständig beheben, sucht das EICiN-RS die Lösung mit der geringsten Grenzwertverletzung.

Kommt es während des Betriebs zu Netzengpässen (Echtzeit; rote Ampelphase), greift das VNA in den Netzbetrieb ein und übernimmt die Netzführung, bis das Netz wieder die grüne Ampelphase erreicht. Während dieser Zeit werden keine Flexibilitätsabrufe des EICiN-RS in den Zellen umgesetzt.

Das Konzept setzt zunächst voraus, dass die Zellen, welche Prognosen und Flexibilitäten übermitteln, „intelligent" ausgestattet sind. D. h., dass diese Zellen über Automatisierungs- und Kommunikationstechnik verfügen ihre Erzeugung und Verbrauch zu prognostizieren, zu optimieren und die Flexibilität zu ermitteln sowie die Daten zu übertragen und Abrufe zu empfangen und umzusetzen. Es ist jedoch nicht davon auszugehen, dass jede Zelle in einem NS-ON zu einem Stichtag intelligent ausgestattet sein wird bzw. alle ASN in den Zellen an diesem System der Flexibilitätsbereitstellung teilnehmen wollen. Für die Ermittlung von Netzengpässen ist zunächst die Prognose entscheidend. Diese könnte daher für konventionelle („unintelligente“) Zellen der Netzbetreiber oder der jeweilige Lieferant der Zelle übernehmen und dem Netzbetreiber mitteilen. Dadurch wird die Funktion des EICiN-RS in einem Hybriden-System gewährleistet (Anforderung 7). Flexibilitäten werden jedoch nur in intelligenten Zellen umgesetzt. Grundsätzlich ist das Vorhandensein von intelligenten Zellen mit Flexibilitätsbereitstellung Voraussetzung für die Funktionalität des EICiN-RS.

Abbildung 1 zeigt den Systemaufbau nach dem EICiN-Regelungskonzept.

Fazit und Ausblick Das Konzept des RS setzt den zellularen Ansatz des VDE um und hält die gesetzten Rahmenbedingungen ein. Grundsätzlich ist das Konzept unabhängig von der Art der Zellen und der Spannungsebene. So lässt sich das Konzept auch auf höhere Spannungsebenen des zellularen Ansatzes anwenden, wenn beispielsweise NS-ON Zellen zusammen mit größeren Gewerbe- bzw. kleineren Industriezellen (Zellebene 4) in einer Mittelspannungsnetzzelle (Zellebene 3) zusammengefasst werden. Die Übertragung auf höhere Spannungsebenen wurde und wird im Forschungsprojekt ElCiN nicht näher untersucht. 


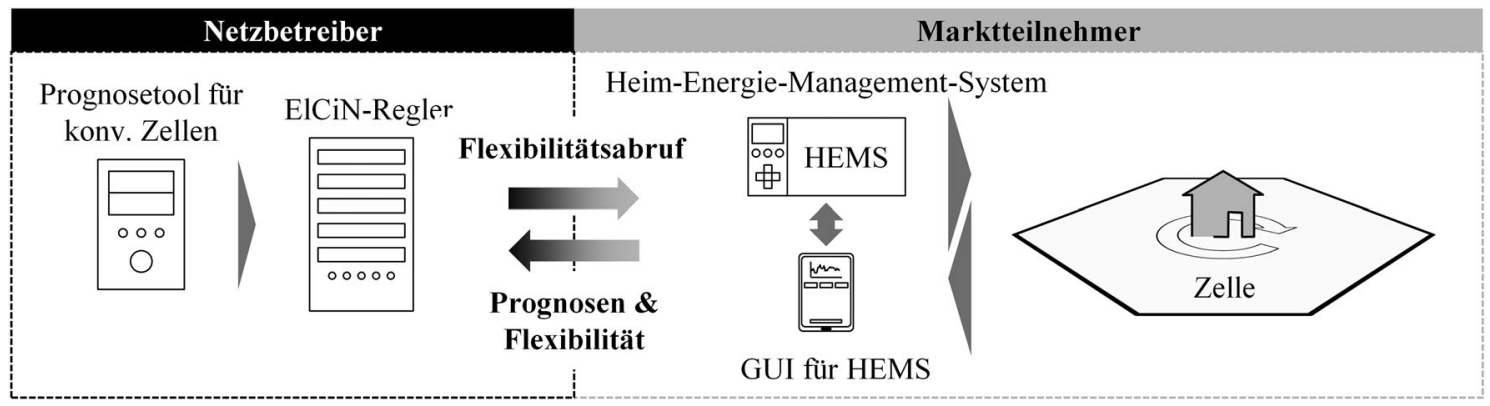

Abb. 1. Darstellung des EICiN-Regelungskonzeptes

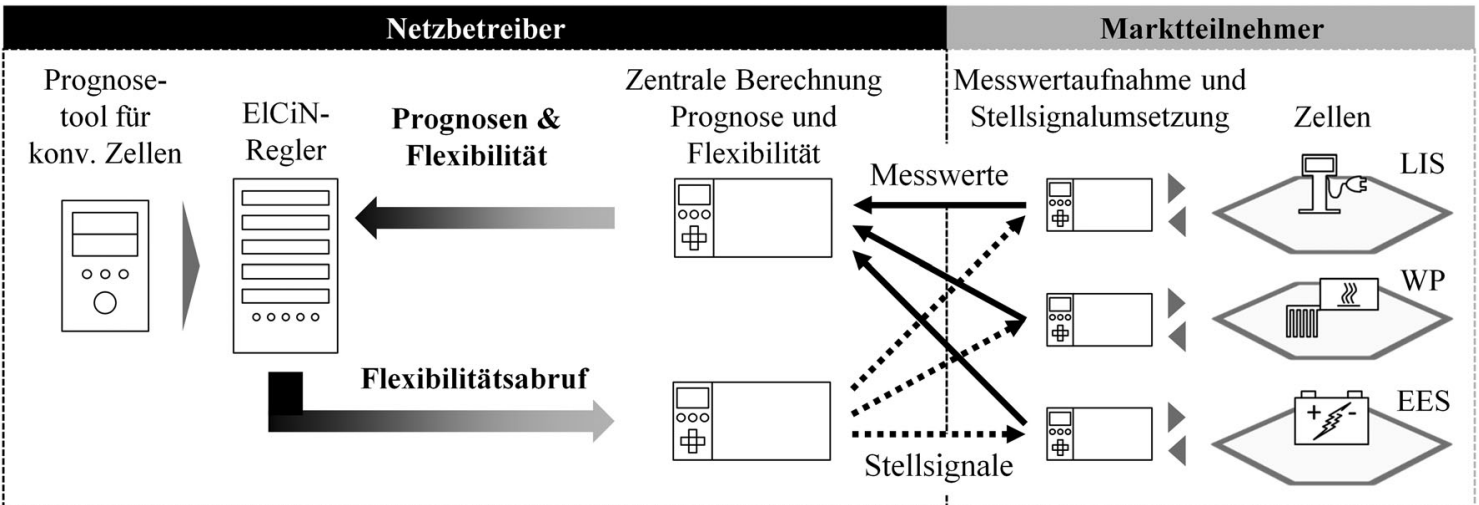

Abb. 2. ElCiN-Systemaufbau für die Erprobung im Feldtest

\subsubsection{Umsetzung des Konzepts im Feldtest}

Bei Fertigstellung des Regelungskonzepts existierten keine HEMS oder ähnliche Produkte, die die Anforderungen des EICiN-RS umsetzen konnten. Daher wurde für den praktischen Nachweis der Funktionsweise des EICiN-RS der Systemaufbau geändert. Zunächst wurde festgelegt, dass einzelne Aktoren wie LIS, EES und WP einzelne Zellen darstellen. Eine Optimierung von mehreren Aktoren innerhalb einer Zelle, wie es das Konzept grundsätzlich erlaubt, wird hier nicht ermöglicht. Des Weiteren wurden die im Feldtest vorhandenen Aktoren analysiert und Prognose- und Flexibilitätsalgorithmen entwickelt, welche die notwendigen Prognosen und Flexibilitätsangebote erzeugen und an das EICiN-RS übermitteln. Zudem sieht der Feldtestaufbau nicht vor, dass diese Algorithmen in der Zelle selbst laufen und die entsprechenden Daten an das EICiN-RS übermitteln, sondern dass die notwendigen Messdaten und Anlageneinstellungen durch den Netzbetreiber und seinen Partnerfirmen vor Ort aufgenommen werden und dann an das System, auf dem auch das EICiN-RS läuft, übermittelt werden. Hier wird zunächst zentral in einem speziellen Tool, welches zur Bestimmung der Prognose und Flexibilität eines jeden Aktors entwickelt wurde, die jeweilige Prognose und Flexibilität im Feldtest ermittelt. Von diesem Tool gehen die entsprechenden Werte dann an das EICiN-RS. Die vom EICiN-RS berechneten Flexibilitätsabrufe werden in Stellwerte umgewandelt und an die Systeme des Netzbetreibers bzw. deren Partnerfirmen übergeben und vor Ort umgesetzt. Abbildung 2 zeigt den entsprechenden Feldtestaufbau.

Trotz der notwendigen Konzeptanpassungen für den Feldtest kann die Funktion des EICiN-Regelungskonzeptes untersucht und bestätigt werden.

\subsubsection{Simulationsbeispiel des Konzepts}

Das EICiN-Regelungskonzept wurde bereits in mehreren Simulationsberechnungen auf Funktionalität geprüft. Beispielhaft sollen Simulationsergebnisse eines realen (Feldtest-)Netzes mit 84 Hausanschlüssen in einem Einfamilienreihenhaus geprägten Quartier dargestellt werden. Das Netz besteht aus einem Transformator mit einer Nennleistung von 250 kVA und 4 Abgängen. Im Netz sind 8 PV Anlagen mit einer Gesamtleistung von 36 kWp, ein EES mit 6,4 kW Ladeleistung und 6,4 kWh Speicherkapazität sowie 3 LIS mit je 22 kW maximaler Ladeleistung verbaut. Der EES und die LIS können als Flexibilität genutzt werden, sofern diese Flexibilität dem EICiN-RS im jeweiligen Zeitschritt anbieten.

Abbildung 3 zeigt die Simulationsergebnisse für das Netz in den Abendstunden eines Sommertages für einen Zeitraum von $120 \mathrm{Mi}-$ nuten. Auf Basis der übergebenen Prognosen aus der Simulationsumgebung detektiert das ElCiN-RS für die zweite und dritte Regelungsperiode (15-30 und 30-45 Minuten) jeweils einen Netzengpass (grau hinterlegte Zeitbereiche in Abb. 3), hier eine Leitungsüberlastung (unter fiktiven Belastungsgrenzen) der gleichen Leitung. In diesen Zeitschritten bieten die Aktoren im Netz dem ElCiN-RS Flexibilitäten zur Lastreduzierung (positive Flexibilität) von $44 \mathrm{~kW}$ bzW. 35 kW an (siehe Abb. 3 (a)). Zur Behebung der Netzengpässe aktiviert das EICiN-RS für die zweite Regelungsperiode zunächst 1,9 kW Wirkleistung und für die dritte Periode $6,8 \mathrm{~kW}$ Wirkleistung positive Flexibilität zur Behebung des Netzengpasses. Abbildung 3 (b) zeigt den Residuallastverlauf des Netzes. Es ist deutlich zu erkennen, wie durch die Aktivierung der Flexibilität die Gesamtlast des Netzes im Zeitraum der Regelung unter der Residuallast ohne Reglereingriff liegt. Durch Abruf der Flexibilität und den individuellen (Optimierungs-)Vorgaben der ASN sinkt die Flexibilitätsbereitstellung mit Abruf von Flexibilität (Abb. 3 (a)). Des Weiteren sind in Abb. 3 (b) 
(a)

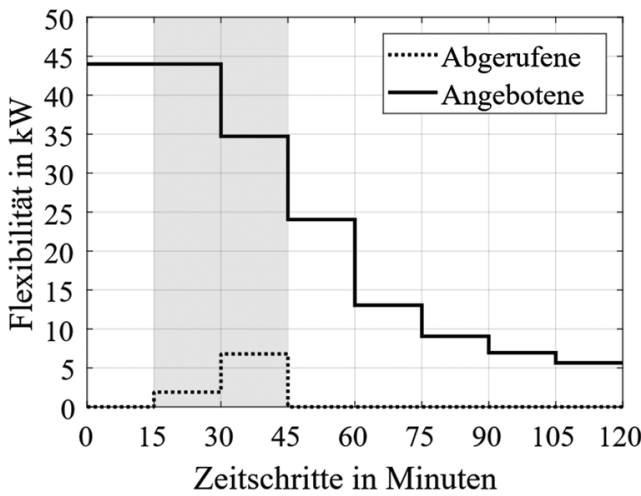

(b)

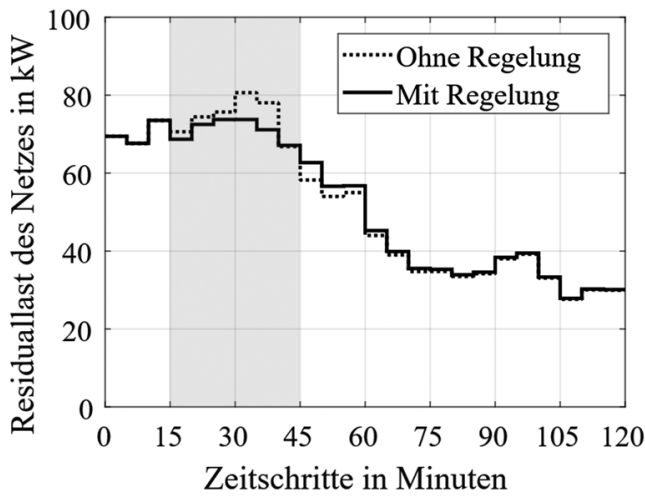

Abb. 3. Ergebnisse der Simulation - (a) dem EICiN-RS angebotene und abgerufene Gesamtflexibilität - (b) der Residuallastverlauf des NS-ON mit und ohne Regelung des EICiN-RS in 5-Minuten Auflösung
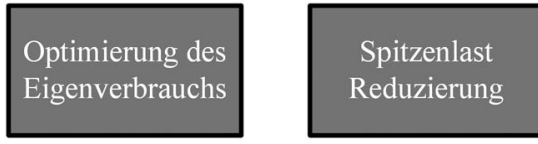

Optimierung 24 Stunden vor Energielieferung
Fahrplan zum

Spotmarkt

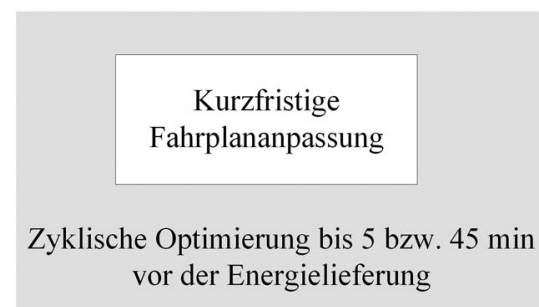

Abb. 4. Wirtschaftliche Optimierung des Gebäudes im InFlex-Konzept

die Nachholeffekte der flexiblen Anlagen nach dem Reglereingriff zu erkennen. Hier liegt die sich ergebene Residuallastkurve über der Kurve ohne Reglereingriff. Beides liegt in den individuellen Optimierungszielen der Flexibilitäten der ASN begründet.

\subsection{InFleX}

\subsubsection{Einleitung und Ziele}

Aufgrund des steigenden Anteils an dezentralen Energieerzeugern durch die Energiewende, steigt der Bedarf an steuerbarer Aktorik im elektrischen Verteilnetz [16]. Das Ziel des InFleX Projektes ist die Integration gebäudetechnischer Flexibilitäten und kombinierter LIS in ein dezentrales VNA. Vor diesem Hintergrund wird ein Gebäuderegler (GR) entwickelt, welcher die gebäudetechnischen Flexibilitäten von Nichtwohngebäuden ermittelt und einem VNA zur Verfügung stellt.

Die flexiblen Komponenten von Nichtwohngebäuden wurden vor allem in den Bereichen Wärme und Kälte identifiziert, da Gebäude aufgrund ihrer thermischen Masse in der Lage sind, thermische Energie zu speichern [17]. Dieses Speicherpotenzial ermöglicht einen flexiblen Betrieb der Wärme- und Kälteerzeugungsanlagen. Weiterhin wurden Lüftungssysteme als mögliche flexible Anlage identifiziert, da sich die Veränderung der Luftqualität träge verhält [18]. Bei der Steuerung dieser Anlagen ist zu beachten, dass Grenzen für Komfortvariablen im Gebäude eingehalten werden müssen. Als Komfortvariablen gelten unter anderem die Raumtemperatur und die $\mathrm{CO}_{2}$-Konzentration in der Luft. In den Normen DIN EN 16798-1 und DIN EN 16798-3 ist definiert, inwieweit sich die Raumluftqualität in Räumen verändern darf, ohne dass der Gebäudenutzer diese Veränderung als negativ empfindet.

Andere flexible Komponenten von Gebäuden sind PV-Anlagen, welche oft in Kombination mit EES eingesetzt werden. Die PVAnlage selbst kann abgeregelt oder die Energie in dem EES gespei- chert werden. Der EES kann auch entladen werden, um die netzbezogene Last zu reduzieren [19].

Ein ähnliches Flexibilitätspotential ergibt sich für die LIS. Dort kann die Ladeleistung reduziert werden, sollte der Netzzustand dies erfordern [20]. Um Komfortvariablen nicht zu verletzen und die Anlagen im Gebäude effizient zu steuern, muss ein GR entwickelt werden, welcher in der Lage ist, diese Aufgaben und Anforderungen sicher und zuverlässig umzusetzen.

\subsubsection{Aufgaben des Gebäudereglers}

Damit die Installation des GRs auch wirtschaftliche Anreize für den Gebäudebetreiber setzt, sollen die verfügbaren Flexibilitäten im Gebäude dazu genutzt werden, das Gebäude so kostengünstig wie möglich zu betreiben. Das Konzept dafür ist in Abb. 4 dargestellt.

Das Konzept wird mit einem Optimierer umgesetzt, welcher im Rahmen des Projektes entwickelt wird. Aufgrund des nichtlinearen Verhaltens des Gebäudes und des Fehlens eines genauen Gebäudemodells kann die Optimierung des Gebäudebetriebsplans mithilfe metaheuristischer Methoden durchgeführt werden [21]. Insbesondere genetische Algorithmen sind von Interesse, da sie im Vergleich zu linearen Methoden suboptimale, aber akzeptable Lösungen in kürzerer Zeit liefern können [22]. Dieses Konzept basiert auf der Evolution einer Population von Fahrplänen für die Sollwerte der Gebäudeanlage, die sich nach jeder Iteration (Generation) rekombiniert und mutiert, auf der Suche nach einer neuen Population, welche die Betriebskosten des Gebäudes reduziert [22]. Fahrpläne, die zu Komfortverletzungen führen, sollen hier ebenfalls ausgeschlossen werden.

Eine der Aufgaben, die der Optimierer berücksichtigt, ist die Optimierung des Eigenverbrauchs. Hierfür wird der Leistungsbezug der flexiblen Anlagen in Zeiträume mit hoher Eigenenergieerzeugung verschoben. Im nächsten Schritt werden Leistungsspitzen des Gebäudes geglättet, damit der Leistungspreis [23] den die Gebäudebe- 


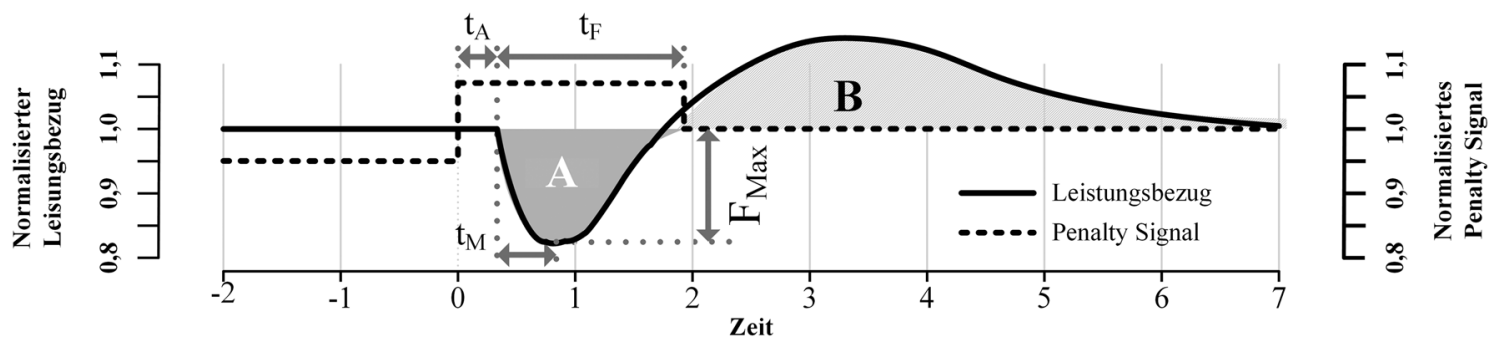

Abb. 5. Flexibilitätsfunktion zur Bestimmung der Gebäude-Flexibilitäten nach Annex 67 [24]

treiber zahlen müssen, minimiert wird. Die dann noch zur Verfügung stehenden Flexibilitäten werden an der Strombörse (Spotmarkt) vermarktet [23].

Am Ende dieser Optimierung wird ein Fahrplan (FP) für das Gebäude definiert, welcher Sollwerte für die flexiblen Anlagen bereitstellt. Sollten kurzfristige Probleme in dem versorgenden elektrischen Verteilnetz auftreten und das Gebäude von der VNA eine Anfrage zur Leistungsanpassung erhalten oder sich mögliche Gewinne durch eine kurzfristige Vermarktung von Flexibilitäten ergeben, muss der Optimierer reagieren. Der ursprüngliche FP wird verlassen und ein neuer FP wird definiert, sodass die Leistungsanpassung der VNA umgesetzt wird oder die vermarkteten Flexibilitäten nicht mehr genutzt werden.

Damit der Optimierer einen FP für das Gebäude erstellen kann, muss dieser prognostizieren, wie sich das Gebäude bei einer Steuerung der flexiblen Anlagen verhält und wie sich dies auf die Komfortvariablen des Gebäudes auswirkt. Das Ziel der Prognose ist, dass sich keine negativen Einflüsse auf die Personen im Gebäude ergeben. Dafür wird eine Künstliche Intelligenz (KI) aufgebaut und mit historischen Daten des Gebäudes trainiert, sodass das Gebäudeverhalten prognostiziert werden kann. Bei der KI handelt es sich hier um ein Neuronales Netz. Dieses ist dem menschlichen Gehirn nachempfunden. Dabei werden Neuronen unterschiedlich stark miteinander verknüpft. So können in den Eingabedaten natürlich Muster erkannt werden und den entsprechenden Ausgabedaten zugeordnet werden. Beispielsweise kann so der Einfluss von Uhrzeit, Tag Typ, Raumbelegung und Jahreszeit der Gebäudetemperatur zugeordnet werden. Mithilfe dieser KI kann auch die Flexibilität des Gebäudes evaluiert werden. Die Flexibilität des Gebäudes für einen bestimmten Zeitpunkt wird hierbei mit der Flexibilitätsfunktion (FF) bestimmt, welche ein Ergebnis des Annex 67 ist [24]. Diese ist in Abb. 5 dargestellt

Die FF beschreibt, wie ein flexibles Gebäude auf ein Penalty Signal, welches ein Strompreis sein könnte, reagieren kann. Sollte der Strompreis steigen, welcher in Abb. 5 das Penalty Signal ist, könnte das Gebäude für eine gewisse Zeit seinen Leistungsbezug senken (dunkelgrauer Bereich). Danach müsste jedoch der Leistungsbezug des Gebäudes für eine gewisse Zeit erhöht werden, damit der ursprüngliche Gebäudezustand wiederhergestellt werden kann (hellgrauer Bereich in Abb. 5). Die FF liefert wichtige Größen, welche an die VNA weitergegeben werden. Die Größe $t_{A}$ gibt die Zeit bis zur ersten Reaktion der Gebäudekomponenten auf das Penalty Signal an. Die Größe $t_{M}$ gibt die Zeit an, welche benötigt wird, bis die ma-

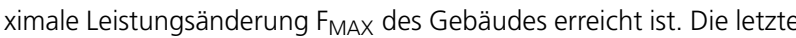
Zeitgröße ist $t_{F}$, welche Angibt, wie lange das Gebäude seine Leistung reduzieren kann.

Die beiden Größen A und B geben die Energie an, welche vom Gebäude nicht bezogen (A) und welche zur Erreichung des ursprünglichen Gebäudezustands mehr bezogen wurde (B).
Durch die vom GR bereitgestellten FF kann das VNA relevante Informationen erhalten, wie z. B. die Leistung, welche das Gebäude mittels dessen flexiblen Anlagen erhöhen oder verringern kann, sowie die Dauer dieses Betriebszustands. Diese Informationen können es dem VNA erleichtern, das Demand Response in einer kritischen Netzsituation zu verwalten und zu steuern. Zusätzlich liefert die FF Informationen über die Veränderung des Energieverbrauchs des Gebäudes nach der Kontrolle.

Die FF wird mithilfe des Optimierers und der KI bestimmt. Der Optimierer erhält hierbei die Aufgabe, die maximale Flexibilität des Gebäudes abzurufen, bis Grenzwertverletzungen der Komfortvariablen auftreten und im Anschluss den ursprünglichen Gebäudezustand wiederherzustellen. Die KI gibt auf diese Anforderung den Leistungsbezug des Gebäudes an.

\subsubsection{Kommunikationsanforderungen}

Für die sichere Ausführung der oben genannten InFleX-GR Funktionalitäten werden in diesem Abschnitt die Kommunikationsanforderungen zwischen den verschiedenen beteiligten Akteuren beschrieben. Diese sind in Abb. 6 dargestellt und auf fünf verschiedenen Ebenen angesiedelt.

Auf der Feldebene befindet sich das zu steuernde Gebäude, das über flexible Komponenten wie Klimaanlagen, LIS, EES und PV verfügt. Diese Komponenten werden in der Regel von einem Gebäudeleitsystem (GLS) über Gebäudekommunikationsprotokolle wie z. B. KNX, BACnet, Modbus gesteuert [25]. Das GLS erhält Informationen von allen im Gebäude installierten Sensoren über Messsignale, welche u. a. Temperatur, Luftqualität und Leistungsbezug enthalten, und steuert die Anlage über Betriebssignale.

Der InFleX-GR liegt in der Controllerebene. Dieser hat die Fähigkeit, die Sollwerte (SW) der flexiblen Gebäudekomponenten basierend auf dessen Betriebs- und Komfortvariablen zu überschreiben. Dies bedeutet, dass der GR die Fähigkeit besitzen sollte, die eingehenden gebäudetechnischen Daten zu deren späterer Analyse in ein einheitliches Format umzuwandeln. Diese Informationen sind Teil der Eingangsparameter des Optimierers und der KI, welche die entsprechenden SW der flexiblen Komponenten definieren. Auch hier ist es erforderlich, dass der GR die berechneten SW in die Sprache der Gebäudeleittechnik übersetzt.

Details zum Zusammenspiel zwischen dem InFleX-GR, dem VNA, dem Energiehändler und den externen Daten werden im Folgenden erläutert.

Auf der Smart Grid / Netz Ebene befindet sich das VNA, welches Aktoren im Netz wie PV, LIS und EES aber zukünftig auch Nichtwohngebäude und konventionelle Haushalte ansteuern kann [26]. Das VNA erkennt die kritische Netzsituationen und sendet Anfragen zur Leistungsreduzierung bzw. -erhöhung an die registrierten und qualifizierten Aktoren (z.B. das Gebäude mit intelligentem InFleXGR), um die Störung zu beheben bzw. präventiv zu vermeiden. 


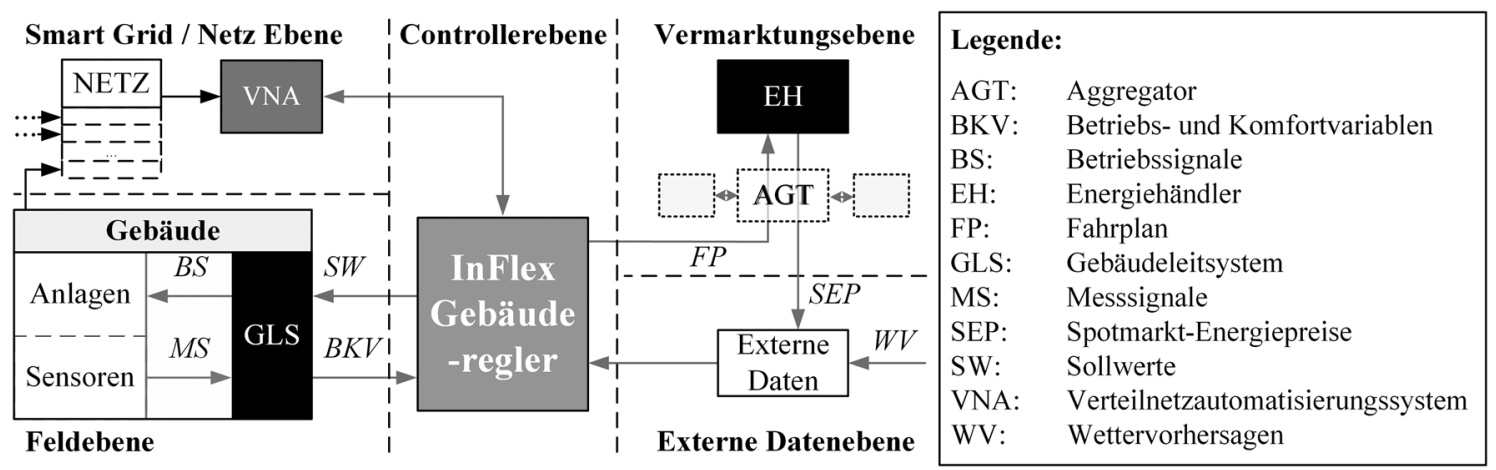

Abb. 6. Kommunikations- und Ebenestruktur für den InFleX-GR

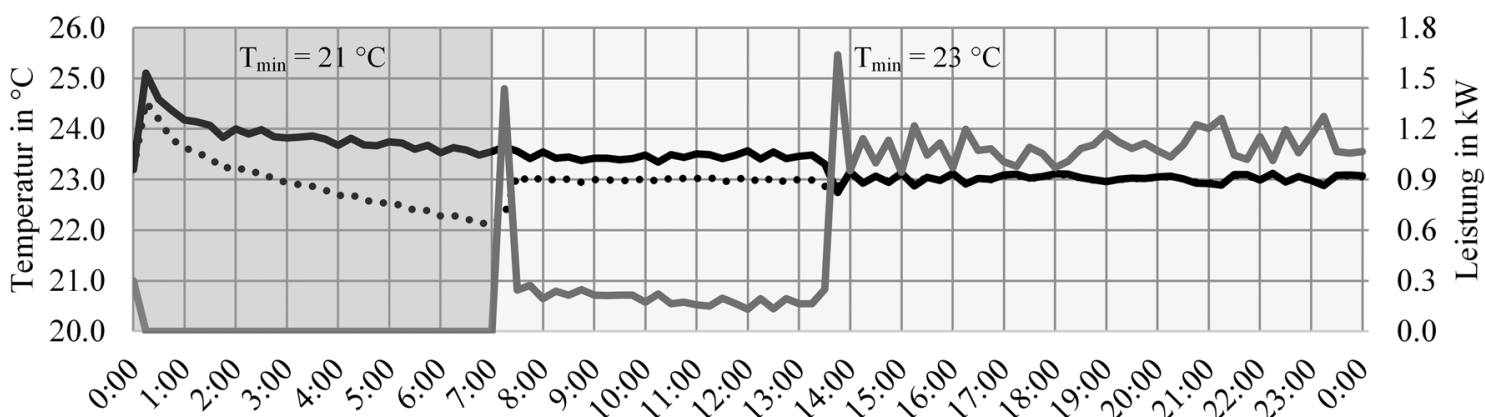

Uhrzeit

•.... Temperatur Zone $1 \quad$ Temperatur Zone $2 \quad$ Heizleistung

\section{Abb. 7. Beispielhafte Steuerung der Komfortvariablen eines Gebäudes zur Bereitstellung von Flexibilitäten}

Das Gebäude sendet alle 15 Minuten Informationen über seine Flexibilität, die über die FF ermittelt wird. Um mit bereits installierten VNAs kommunizieren zu können, wird das Gebäude wie eine PVAnlage behandelt, damit bestehende Schnittstellen für den InFleXGR genutzt werden können [27]. Die Kommunikation zwischen ihnen wird über das IEC 60870-5-104-Protokoll oder alternativ über das Modbus-Protokoll realisiert.

Wie bereits erwähnt, legt der InFleX-GR den FP des Gebäudes in Abwesenheit einer Vorgabe durch das VNA auf Basis der SpotmarktEnergiepreise fest. Er kann z.B. die Informationen aus dem Spotmarkt über eine externe Datenschnittstelle entnehmen, um einen optimierten FP zu definieren und diesen direkt an den Energiehändler oder einen Aggregator zu senden. Mit welchem von beiden kommuniziert wird, hängt von der gesamten flexiblen Leistung des Gebäudes ab [28]. Dieser Datenaustausch wird wie in bereits durchgeführten Projekten durch das IEC 60870-5-104 Protokoll mittels einer Vermarktungsplattform in der Vermarktungsebene erreicht [23].

Um die Genauigkeit der KI zur Prognose des Gebäudezustandes zu verbessern, werden Wettervorhersagen von Online-Plattformen berücksichtigt, die über das Ethernet Protokolle mithilfe von HTMLAbfragen bereitgestellt werden (unter externer Datenebene in Abb. 6). Diese können vom InFleX-GR zur Schätzung der bereitgestellten Energie der PV-Anlage und zur Optimierung der Speicherung der thermischen Energie im Gebäudes genutzt werden.

Das Gebäude wird in der Regel nach dem für den Energiehandel festgelegten FP betrieben. Der InFleX-GR hat die Aufgabe, die definierten SW in einer gebäudetechnischen Sprache an das GLS zu senden. Gleichzeitig wird überwacht, dass dieser Vorgang keine Komfortanforderungen verletzt, und wenn dies der Fall ist, wird der
GR den FP ändern und an kurzfristigen Märkten den Energiebedarf nachjustieren.

Andererseits sendet der InFleX-GR bei Vorliegen einer Anforderung vom VNA die SW an das GLS, sodass die Anforderungen der VNA erfüllt werden. Dies führt auch zu einer Neujustierung des FPS am kurzfristigen Spotmarkt.

\subsubsection{Flexibilitätsabruf durch Gebäuderegler}

In diesem Abschnitt wird eine beispielhafte Regelung eines Gebäudes vorgestellt. Dafür wurde in einem Gebäudesimulationsprogramm ein Gebäude mit zwei Zonen (Räumen) erstellt. Die Temperatur dieser zwei Zonen wurde im Anschluss von einem einfachen GR gesteuert. Die Ergebnisse dieser Steuerung sind in Abb. 7 dargestellt.

Das Simulationsprogramm erfordert eine Vorsimulation, um Ausgangsbedingungen mit geringen Abweichungen zu erhalten. Das abnehmende Temperaturverhalten in beiden Zonen in den frühen Morgenstunden ist auf die kalten Außenlufttemperaturen zurückzuführen, die zu einer Abkühlung der Räume führt, da die Heizung aufgrund keiner vorliegenden Temperaturverletzungen abgeschaltet wird.

Da Zone 1 im Gegensatz zu Zone 2 ein großes Fenster besitzt, kühlt diese Zone schneller aus als Zone 2 .

Um 7 Uhr wurde ein starker Einspeisefall im Verteilnetz simuliert, weshalb die VNA vom GR eine erhöhte Leistungsaufnahme gefordert hat. Deshalb wurde der Sollwert für die minimale Temperatur in beiden Zonen ab 7:00 Uhr (hellgrauer Bereich in Abb. 7) auf $23^{\circ} \mathrm{C}$ angehoben. Aus diesem geänderten Sollwert resultiert eine Flexibilität von ungefähr 1,4 kW, welche dem VNA zur Verfügung 
gestellt werden kann. Nachdem die Temperatur in Zone 1 auf die neue minimale Temperatur gestiegen ist, reduziert sich auch die benötige Heizleistung wieder, da der Wert nur noch gehalten werden muss. Um ungefähr 14:00 Uhr fällt die Temperatur in Zone 1 und 2 noch einmal auf unter $23^{\circ} \mathrm{C}$ woraus ein weiterer Leistungssprung der Heizleistung resultiert. Diese plötzliche Temperaturabsenkung in beiden Zonen folgt aus einer Mischung von einer wesentlich kälteren Außentemperatur und aus einer Belegungsänderung zu diesem Zeitpunkt. Daraus ergibt sich auch die erhöhte Heizleistung zur Temperaturhaltung.

\section{Synergien zwischen ElCiN und InFleX}

Wie bereits in der Einleitung beschrieben, ist eine Steigerung an neuen Aktoren im Verteilnetz wie PV-Anlagen, LIS, EES und WP zu erwarten. Speziell die zusätzlichen Verbraucher belasten in ihrer zu erwartenden Anzahl das Verteilnetz, welches dafür nicht ausgelegt ist, weshalb in Zukunft mehr Netzengpässe zu erwarten sind $[4,5]$. Diese temporären Netzengpässe können durch Nutzung von Flexibilitäten, wie oben beschrieben, behoben werden. Sowohl die im EICiN- als auch im InFleX-Projekt entwickelten Systeme zur Prognose und Flexibilitätsbestimmung sind in der Lage, die Anforderungen an ein System zum marktorientierten Netzengpassmanagement zu erfüllen. Die Systeme EICiN-RS und InFleX-GR sind grundsätzlich miteinander kompatibel, da beide Systeme die notwendigen Daten (Prognose und Flexibilität bzw. Flexibilitätsabruf/Penalty Signal) jeweils zur Verfügung stellen bzw. mit den Daten arbeiten können. Die Systeme ergänzen sich somit zu einem, über alle Zellebenen, intelligenten Gesamtsystem.

Der InFleX-GR wird im Marktbereich (nicht reguliert) eingesetzt und ist unabhängig von der Art der eingesetzten Netzautomatisierung. Der InFleX-GR kann hier sowohl auf reine Steuerungssignale einer klassischen Netzautomatisierung reagieren sowie mit einem marktbasierten Regler (bspw. ElCiN-RS) in einem Gesamtsystem agieren.

Betrachtet man die Prognose für Smart Home Systeme mit Energy Management in Deutschland, so sollen bis 2025 15,31 Mio. Haushalten ein entsprechendes System besitzen [29]. Dies entspricht einer Steigerung von 11,76 Mio. im Vergleich zu 2020 mit 3,55 Mio. Systemen [29]. Bei 41,5 Mio. Haushalten (Stand 2020) [30] entspricht dies einer Abdeckung von 36,9\% in 2025 im Vergleich zu $8,6 \%$ in 2020. Es wird in [29] allerdings nicht definiert, was Smart Home Systeme mit Energy Management konkret bedeutet und welche Systeme dies einschließt und welche nicht.

Unterstellt man, dass es sich bei den prognostizierten Systemen im Allgemeinen um HEMS handelt, welche auch einem für Wohngebäude weiterentwickelten InFleX-GR entsprechen könnten, so könnten bis 2025 nach der oben genannten Prognose 36,9 \% der Haushalte potenziell vollautomatisiert Flexibilitäten anbieten.

Dieses Potenzial kann wiederum das EICiN-RS, welcher sich im regulierten Bereich befindet, nutzen, um mittels der kurzfristigen Prognosen und Flexibilitätsangebote prognostizierte Netzengpässe marktorientiert und vollautomatisiert zu beheben. Für Verteilnetze mit vielen Nichtwohngebäuden wäre eine Kopplung der EICiN- und InFleX-Systeme theoretisch bereits jetzt möglich.

\section{Fazit und Ausblick}

In diesem Artikel wurden zwei Add-on Systeme für klassische VNA vorgestellt. Das EICiN-System, welches sich im regulierten Bereich befindet, führt mittels Prognosen und Flexibilitäten einzelner Zellen ein dynamisches und marktorientiertes Netzengpassmanagement durch. Das InFleX-System führt eine intelligente Gebäudeautomation mit Flexibilitätsbereitstellung für VNA durch.
Ein sich ergebenes Gesamtsystem aus EICiN-RS und InFleX-GR entspricht dabei dem EICiN-Projekt entwickelten Gesamtkonzept und setzt dabei den zellularen Ansatz des VDE um.

Zu den rechtlichen Rahmenbedingungen lässt sich an dieser Stelle sagen, dass dem EICiN-RS für einen vollautomatisierten Flexibilitätsmarkt aktuell die gesetzliche Grundlage für eine Umsetzung in der Praxis fehlt. Die Untersuchung der notwendigen Ausgestaltung der rechtlichen Rahmenbedingungen und ggf. einen Transformationspfad ist jedoch nicht Teil des ElCiN-Projektes.

Soll ein vollautomatisierter Flexibilitätsmarkt zur Netzengpassbehebung implementiert werden, müssen sowohl das EICiN-System als auch das InFleX-System weiterentwickelt und zusammengeführt werden.

Für das EICiN-System stehen hier vor allem die Festlegung eines Kommunikationsprotokolls sowie die notwendigen rechtlichen Rahmenbedingungen für den Betrieb eines solchen Systems im Mittelpunkt.

Der Weiterentwicklungsschwerpunkt für das InFleX-System ist wiederum die Implementierung des Gebäudereglers speziell für Wohngebäude.

Beide Systeme sowie ihre Kombination in einem gemeinsamen Gesamtsystem besitzen nach Meinung der Autoren das Potenzial einen wichtigen Beitrag in Bezug auf die Herausforderungen der Energiewende auf Verteilnetzebene zu leisten.

\section{Danksagung}

Diese Veröffentlichung wurde im Rahmen der Forschungsprojekte Electric City Neuss (kurz: EICiN) und Integration gebäudetechnischer Flexibilitäten und kombinierter Fahrzeugladeinfrastruktur in ein dezentrales Verteilnetzautomatisierungssystem (kurz: InFleX) erstellt.

EICiN und InFleX sind aus dem Europäischen Fonds für regionale Entwicklung (EFRE) 2014-2020 "Investitionen in Wachstum und Beschäftigung" und dem Land Nordrhein-Westfalen geförderte Forschungsvorhaben.

Die Gesamtfördersumme von ElCiN beträgt 1,26 Mio. Euro und ist für drei Jahre im Zeitraum von Juni 2018 bis Mai 2020 angesetzt.

Die Gesamtfördersumme von InFleX beträgt 1,11 Mio. Euro und ist ebenfalls für drei Jahre im Zeitraum von November 2019 bis Oktober 2022 angesetzt.

\section{(2) EFRE.NRW \\ Ministerium für Wirtschaft, Innovation, Digitalisierung und Energie des Landes Nordrhein-Westfalen} Investitionen in Wachstum und Beschäftigung

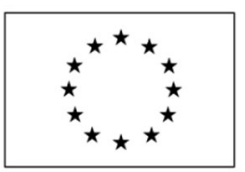

$$
\begin{aligned}
& \text { EUROPÄISCHE UNION } \\
& \text { Investition in unsere Zukunft } \\
& \text { Europäischer Fonds } \\
& \text { für regionale Entwicklung }
\end{aligned}
$$

Funding Note Open Access funding enabled and organized by Projekt DEAL.

Hinweis des Verlags Der Verlag bleibt in Hinblick auf geografische Zuordnungen und Gebietsbezeichnungen in veröffentlichten Karten und Institutsadressen neutral.

Open Access Dieser Artikel wird unter der Creative Commons Namensnennung 4.0 International Lizenz veröffentlicht, welche die Nutzung, Vervielfältigung, Bearbeitung, Verbreitung und Wiedergabe in jeglichem Medium 
und Format erlaubt, sofern Sie den/die ursprünglichen Autor(en) und die Quelle ordnungsgemäß nennen, einen Link zur Creative Commons Lizenz beifügen und angeben, ob Änderungen vorgenommen wurden. Die in diesem Artike enthaltenen Bilder und sonstiges Drittmaterial unterliegen ebenfalls der genannten Creative Commons Lizenz, sofern sich aus der Abbildungslegende nichts anderes ergibt. Sofern das betreffende Material nicht unter der genannten Creative Commons Lizenz steht und die betreffende Handlung nicht nach gesetzlichen Vorschriften erlaubt ist, ist für die oben aufgeführten Weiterverwendungen des Materials die Einwilligung des jeweiligen Rechteinhabers einzuholen. Weitere Details zur Lizenz entnehmen Sie bitte der Lizenzinformation auf http://creativecommons.org/licenses/by/4.0/deed.de.

\section{Literatur}

1. Bundesministerium für Umwelt (2020): Naturschutz und nukleare Sicherheit und www.bmu.de, Klimaschutz in Zahlen: Fakten, Trends und Impulse deutscher Klimapolitik Ausgabe 2020. [Online]. Verfügbar unter: https://www.bmu.de/fileadmin/Daten_ BMU/Pools/Broschueren/klimaschutz_zahlen_2020_broschuere_bf.pdf.

2. Bundesministerium für Umwelt Naturschutz und nukleare Sicherheit und www.bmu. de, Klimaschutzplan 2050 - Klimaschutzpolitische Grundsätze und Ziele der Bundesregierung. [Online]. Verfügbar unter: https://www.bmu.de/fileadmin/Daten_BMU/ Download_PDF/Klimaschutz/Klimaschutzplan_2050_bf.pdf.

3. Schermeyer, H., Studer, M., Ruppert, M., Fichtner, W. (2017): Understanding distribution grid congestion caused by electricity generation from renewables. In C. Derksen C. Weber (Hrsg.), IFIP advances in information and communication technology, smart energy research. At the crossroads of engineering, economics, and computer science (S. 78-89). Cham: Springer. https://doi.org/10.1007/978-3-319-66553-5_6.

4. Richardson, P., Flynn, D., Keane, A. (2010): Impact assessment of varying penetrations of electric vehicles on low voltage distribution systems. In Energy society general meeting, Minneapolis, MN (S. 1-6). https://doi.org/10.1109/PES.2010.5589940.

5. Schwalbe, R., Häusler, M., Stifter, M., Esterl, T. (2017): In Market driven vs. grid supporting heat pump operation in low voltage distribution grids with high heat pump penetration - an Austrian case study, Rotterdam, Netherlands, Juni 2017 [Online]. Verfügbar unter: http://hpc2017.org/wp-content/uploads/2017/05/0.2.3.1 Market-driven-vs.-grid-supporting-heat-pump-operation.pdf. (Zugriff am: 22. April 2021).

6. Röhrenbeck, S. (2019): Wärmepumpen und Speichersysteme als Flexibilität im Kontex des Zellularen Ansatzes. Dissertation, Elektrotechnik und, Informationstechnik, Technische Universität Kaiserslautern, Technische Universität Kaiserslautern.

7. BDEW (2017): In Konkretisierung des Ampelkonzepts im Vertleiungsnetz: Diskussionspapier, Berlin: BDEW. 10. Feb. 2017 [Online]. Verfügbar unter: https://www. bdew.de/media/documents/20170210_Konkretisierung-Ampelkonzept-Smart-Grids. pdf. (Zugriff am: 15. Juni 2021).

8. VDE Verband der Elektrotechnik Elektronik Informationstechnik e.V. (2019): Zellulares Energiesystem: Ein Beitrag zur Konkretisierung des zellularen Ansatzes mit Handlungsempfehlungen. Fachbeitrag, Frankfurt am Main, Ma 2019. [Online]. Verfügbar unter: https://www.vde.com/resource/blob/1884494/ 98f96973fcdba70777654d0f40c179e5/studie---zellulares-energiesystem-data.pdf. (Zugriff am: 15. Juni 2021)

9. ETG Task Force Grundsätzliche Auslegung neuer Netze, Der Zellulare ANsatz: Grundlage einer erfolgreichen, regionenübergreifenden Energiewende, Frankfurt am Main, Juni 2015.

10. Mohaupt, F., Macht, L., Dede und, C., Gährs, S. (2018): In Mögliche Akzeptanzfaktoren für Flexibilitäten im Energiesystem: Eine literaturbasierte Analyse im Rahmen von Arbeitspaket 8 im Projekt Designnetz, Berlin, Jan. 2018

11. SEDC Smart Energy Demand Coalition (2016): In Energy demand coalition, empowering residential and SME consumers, Brüssel: White Paper. Okt. 2016. [Online]

\section{Autoren}

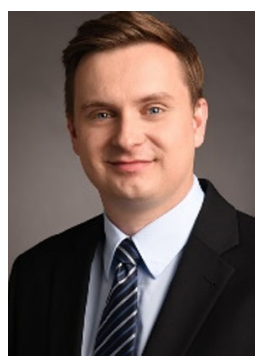

\section{Michael Becker}

wurde 1986 in Dormagen, Deutschland, geboren. Nach dem Abschluss des Studiums des Maschinenbaus 2015 an der Technischen Hochschule Köln war er als Unternehmensberater tätig. Seit 2018 ist er Wissenschaftlicher Mitarbeiter an der Bergischen Universität Wuppertal am Lehrstuhl für Elektrische Energieversorgungstechnik. Seine Forschungsschwerpunkte sind die intelligente Flexibilitätsbereitstellung von Sektorenkopplungsanlagen sowie de-
Verfügbar unter. https://smarten.eu/wp-content/uploads/2016/10/SEDC-White-PaperEmpowering-Residential-and-SME-Consumers.pdf

12. Hermanns, J., et al. (2019): Preparation of a field test to evaluate a local flexibility market as a smart grid add-on. In D. Schulz (Hrsg.), NEIS 2019: conference on sustainable energy supply and energy storage systems, Hamburg, 19-20 September 2019. Berlin: VDE VERLAG GMBH

13. Vaillant (2019): Planungsinformation Wärmepumpen. Holzminden: Vaillant Deutschland $\mathrm{GmbH} \&$ Co. KG.

14. Kotthaus, K., et al. (2019): Local flexibility markets: an economic solution for the upcoming influence of electrical charging station penetration: Madrid. In 25th international conference on electricity distribution CIRED, Madrid, 3-6 June 2019, Liège, Belgium. https://doi.org/10.34890/1042

15. Kotthaus, K., et al. (2020): In New opportunities for congestion management and grid planning due to decentralized flexibility: CIRED 2020, workshop, Berlin, 4-5 June 2020.

16. Aduda, K. O., Labeodan, T., Zeiler, W., Boxem, G., Zhao, Y. (2016): Demand side flexibility: potentials and building performance implications. Sustain. Cities Soc., 22, 146-163. https://doi.org/10.1016/j.scs.2016.02.011.

17. Hurtado, L. A., Rhodes, J. D., Nguyen, P. H., Kamphuis, I. G., Webber, M. E. (2017): Quantifying demand flexibility based on structural thermal storage and comfort management of non-residential buildings: a comparison between hot and cold climate zones. Appl. Energy, 195, 1047-1054. https://doi.org/10.1016/..apenergy.2017.03. 004

18. Penman, J. M., Rashid, A. (1982): Experimental determination of air-flow in a naturally ventilated room using metabolic carbon dioxide. Build. Environ., 17(4), 253-256. https://doi.org/10.1016/0360-1323(82)90017-8

19. Salpakari, J., Lund, P. (2016): Optimal and rule-based control strategies for energy flexibility in buildings with PV. Appl. Energy, 161, 425-436. https://doi.org/10.1016/ j.apenergy.2015.10.036.

20. Uhlig, R. (2017): Nutzung der Ladeflexibilität zur optimalen Systemintegration von Elektrofahrzeugen. Berlin: epubli.

21. Shrivastava, P., Dhingra, S. L., Gundaliya, P. J. (2002): Application of genetic algorithm for scheduling and schedule coordination problems. ATR, 36(1), 23-41. https://doi. org/10.1002/atr.5670360103.

22. Ramos Ruiz, G., Lucas Segarra, E., Fernández Bandera, C. (2019): Model predictive control optimization via genetic algorithm using a detailed building energy model. Energies, 12(1), 34. https://doi.org/10.3390/en12010034

23. Meese, J. (2018): Dynamische Stromtarife zur Erschließung von Flexibilität in Industrieunternehmen. Berlin: epubli GmbH.

24. Knotzer, A., Weiss, T., Metzger, A., Kastner, W. (2019): IEA Energie in Gebäuden und Kommunen (EBC) Annex 67: Energie-flexible Gebäude: Arbeitsperiode 2015 - 2019

25. Lauckner, G., Krimmling, J. (2020): In Raum- und Gebäudeautomation für Architekten und Ingenieure, Wiesbaden: Springer

26. Kolokotsa, D. (2016): The role of smart grids in the building sector. Energy Build., 116, 703-708. https://doi.org/10.1016/j.enbuild.2015.12.033.

27. Korotkiewicz, K. (2020): Koordinierte, teilautarke Regelung von Mittelspannungsnetzen unter Einsatz dezentraler Automatisierungslösungen. Berlin: epubli GmbH.

28. Thomsen, J., Schulz, A., Hartmann, N. (2016): Vermarktung von Flexibilitäten im Verteilnetz - Analyse der Akteure und rollen für zukünftige Ge-schäftsmodelle im Verteilnetz. In Symposium Energieinnovation (EnInnov) (S. 1-12). https://doi.org/10.1515/ 9783110924992-003.

29. Statista Smart Home - Anzahl der Haushalte nach Segmenten in Deutschland 2025 | Statista. [Online]. Verfügbar unter. https://de.statista.com/prognosen/801573/ anzahl-der-smart-home-haushalte-nach-segmenten-in-deutschland. (Zugriff am: 21. Juni 2021).

30. Statistisches Bundesamt, Haushalte und Familien - Ergebnisse des Mikrozensus - Fachserie 1 Reihe 3 - 2019. [Online]. Verfügbar unter: https://www.destatis.de/DE/Themen/ Gesellschaft-Umwelt/Bevoelkerung/Haushalte-Familien/Publikationen/DownloadsHaushalte/haushalte-familien-2010300197004.pdf?_blob=publicationFile.

ren Einbindung in Betriebskonzepte von Verteilnetzbetreibern für Netzengpassmanagement. 


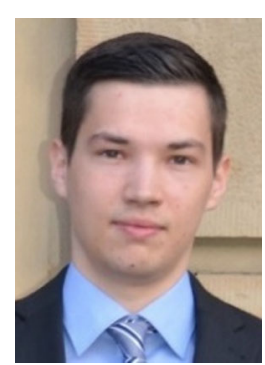

\section{Tobias Müller}

wurde 1995 in Wuppertal, Deutschland, geboren. Im Jahr 2018 hat er seinen Masterabschluss im Studiengang Wirtschaftsingenieurwesen Energiemanagement an der Bergischen Universität Wuppertal gemacht und ist seit 2018 als Wissenschaftlicher Mitarbeiter am Lehrstuhl für Elektrische Energieversorgungstechnik der Bergischen Universität tätig. Sein Forschungsschwerpunkt ist die intelligente Flexibilitätsbereitstellung durch Sektorenkopplungstechnologien und Batteriespeicher für Netzengpassmanagement.

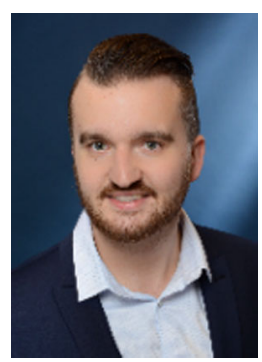

\section{Maximilian Hendrik Forchheim}

wurde 1994 in Wuppertal, Deutschland, geboren. Hat im Jahr 2019 seinen Masterabschluss im Studiengang Elektrotechnik mit dem Schwerpunkt "Renewable Energy" an der Bergischen Universität Wuppertal gemacht und ist seit 2019 als wissenschaftlicher Mitarbeiter am Lehrstuhl für Elektrische Energieversorgungstechnik im Bereich Intelligenter Netze und Systeme an der Bergischen Universität Wuppertal tätig. Sein Forschungsschwerpunkt ist die Integration von Flexibilitäten in ein dezentrales Verteilnetzautomatisierungssystem.

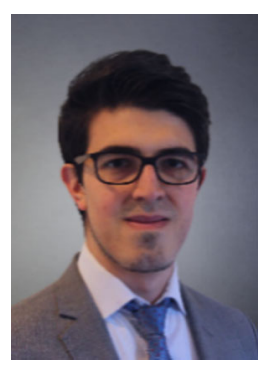

\section{David Cano-Tirado}

wurde 1990 in Bogotá, Kolumbien geboren. Nach Abschluss des Studiums der Elektrotechnik 2013 an der Escuela Colombiana de Ingeniería (Kolumbien) war er dort als wissenschaftlicher Mitarbeiter in den Bereichen Automatisierung, Steuerungstechnik und Energie tätig. Seit 2019 arbeitet er als wissenschaftlicher Mitarbeiter am Lehrstuhl für Elektrische Energieversorgungstechnik im Bereich Intelligenter Netze und Systeme an der Bergischen Universität Wuppertal. Sein Forschungsschwerpunkt ist die Integration von Flexibilitäten in ein dezentrales Verteilnetzautomatisierungssystem.

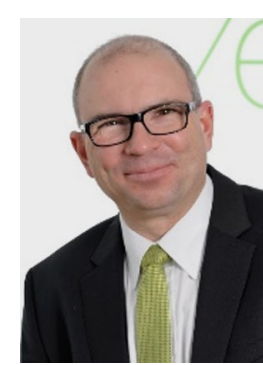

\section{Markus Zdrallek}

wurde 1971 in Darmstadt, Deutschland, geboren. Studium der Elektrotechnik an der Technischen Hochschule Darmstadt, Promotion an der Universität Siegen. Von 2000 bis 2010 war Prof. Zdrallek in verschiedenen leitenden Funktionen des Energieversorgungsunternehmens RWE in Siegen, Brauweiler, Neuss und Warschau tätig - zuletzt als Prokurist der RWE Rhein-Ruhr Netzservice GmbH. Seit April 2010 ist er Professor an der Bergischen Universität Wuppertal, Leiter des Lehrstuhls für Elektrische Energieversorgungstechnik. Zustandsbewertung von Betriebsmitteln, Modellbildung und Optimierung von Netzen und Netzbetrieb sowie "intelligente" Stromnetze für die Energiewende sind die Hauptforschungsgebiete des Lehrstuhls. Gleichzeitig ist Prof. Zdrallek wissenschaftlicher Direktor der Neue Effizienz gemeinnützige GmbH. 